\title{
Simple method for long-term recording of electrocardiogram signals in prawns
}

\author{
Han-Tso Lin ${ }^{1, *}, \#$, Tsen-Chien Chen ${ }^{2, \#}$, Jun-Ping Chang ${ }^{3}$, Zin-Yan Chou ${ }^{3}$, \\ Shian-Yu Chiou ${ }^{3}$, Meng-Li Tsai ${ }^{3}$ \\ ${ }^{1}$ Department of Biotechnology, Ming Chuang University, Taoyuan City 33348, Taiwan \\ ${ }^{2}$ Department of Leisure Management, Minghsin University of Science and Technology, Hsinchu County 30401, Taiwan \\ ${ }^{3}$ Department of Biomechatronic Engineering, National Ilan University, Yilan County 26047, Taiwan
}

\begin{abstract}
This paper presents a novel approach to the long-term recording of electrocardiogram (ECG) signals in prawns, including a 3-lead electrode, a lightweight external wires assembly, a waterproof electrode-wire junction, and a standardized implanting procedure. The proposed low-cost device is easily constructed, even by untrained undergraduate students, using common laboratory materials. Consistent ECG recordings were obtained over a period of $72 \mathrm{~h}$ in experiments on 6 freely moving prawns (Macrobrachium rosenbergii). A subsequent experiment conducted continuously for 38 d revealed that despite gradual attenuation the ECG signals could still be identified (signal-to-noise ratio $\geq 3$ ). The prawns survived implantation and later underwent natural molting, whereupon the electrode was successfully re-implanted. The proposed electrode is a valuable tool by which to gain insight into the long-term physiological state of crustaceans.
\end{abstract}

KEY WORDS: Electrocardiogram · Crustaceans · Giant river prawn · Macrobrachium rosenbergii · Cardiac arrest

\section{INTRODUCTION}

Decapod crustaceans (e.g. crabs, prawns, lobsters, and crayfish) are a diverse group of more than 14000 extant species, many of which are exploited commercially as a source of protein (Deshmukh 2013). Developing a more complete understanding of decapod crustaceans is crucial to the sustainability of this resource.

Crustaceans are sensitive to environmental conditions, including temperature, noise, dissolved oxygen content, photoperiod, and the presence of chemical compounds. Unfortunately, the rigid shell-like exoskeleton of crustaceans makes it difficult to observe physiological responses to external conditions. Researchers have clearly demonstrated a correlation

*Corresponding author: htlin@mail.mcu.edu.tw

\#These authors contributed equally to this work between heartbeat patterns in electrocardiogram (ECG) signals and external stimuli, thereby making it possible to use cardiac activity (e.g. heart rate or heart rate variability) as a proxy for metabolic changes (Ern et al. 2014, Tsai et al. 2019). Numerous researchers have sought to use ECG recordings of crustaceans as a tool to observe underlying physiological functions. Larimer (1962) pioneered the use of real-time ECGs to monitor changes in the cardiac activity of crayfish under various oxygen concentrations. Florey \& Kriebel (1974) recorded ECG patterns from unrestrained crabs to elucidate the effects of temperature, anoxia, and sensory stimulation on heart rate. Through the analysis of ECG recordings, Mickel \& Childress (1982) examined the effects of pressure and temperature on the heart rate of

(C) The authors 2021. Open Access under Creative Commons by Attribution Licence. Use, distribution and reproduction are unrestricted. Authors and original publication must be credited. 
Bythograea thermydron (a deep-sea crab living on hydrothermal vents). Mislin (1966) revealed that light levels could affect the heartbeat of animals. Burnovicz et al. (2009) examined ECG signals from Neohelice granulata (previously known as Chasmagnathus) under flashing lights, bubble-induced noise, and a moving visual danger stimulus present in experiments.

ECG monitoring has revealed some interesting phenomena. Yazawa \& Katsuyama (2001) identified instances of spontaneous repetitive cardiac arrest in freely moving spiny lobsters. Similar research has been used to study the social behavior of crustaceans, such as fighting for territory and/or mating rights. Schapker et al. (2002) used ECG signals to monitor heart rate as an index by which to assess stress associated with social interactions. Listerman et al. (2000) explored the relationship between social interactions and serotonin levels (5-HT) in the hemolymph of crayfish. Based on heart rate, Cooper et al. (2011) monitored the physiological conditions in crustaceans of both sexes during copulation.

In the studies mentioned above, heart activity was characterized from infrared signals in the contraction process of the heart (Ern et al. 2014), changes in the impedance of peripheral components of the heart (Listerman et al. 2000, Schapker et al. 2002, Burnovicz et al. 2009, Cooper et al. 2011), or changes in membrane potential during heart contractions (Larimer 1962, Florey \& Kriebel 1974, Mickel \& Childress 1982, Yazawa \& Katsuyama 2001). Overall, changes in membrane potential present the richest information, in terms of calculating heart rate, indicating the electrical dynamics associated with populations of myocardial cells in various regions of the heart and even physiological or pathological conditions. However, no previous study based on membrane potential has been conducted for extended periods (e.g. several weeks). It is therefore difficult to determine whether those recording methods provide stable results over the long term. Yazawa \& Katsuyama (2001) reported that their approach to ECG recording could be continued for several weeks; however, no mention was made as to the quality of ECG recordings over time.

Furthermore, those articles did not provide a clear description of the position of the electrode leads implanted in the animal's body (i.e. using vague terms, such as cardiac region). The waveforms of the ECG recording vary as a function of the position of the leads on the heart. Irisawa \& Irisawa (1957) demonstrated that the ECG waveforms obtained from the surface of peripheral heart regions differ from those obtained from the mid-dorsal region of the heart. In mammals, every characteristic of the ECG waveform can be attributed to electrophysiological activity. Thus, it is essential that researchers maintain consistency in the ECG recording process.

This paper presents a novel approach to the longterm recording of ECG signals in prawns, including a 3-lead electrode with lightweight external wires and waterproof electrode-wire junctions providing buoyancy. In experiments, the proposed electrode implantation scheme was shown to improve the accuracy of electrode placement, resulting in highly consistent ECG signals between individuals with a signal-tonoise ratio remaining at a readable level even after monitoring for an extended duration. Electrode implantation appears to have only a minor physiological impact on the animals, as indicated by continued normal growth and molting following the operation.

\section{MATERIALS AND METHODS}

\subsection{Preparation of animals for experiments}

Live specimens of the giant river prawn Macrobrachium rosenbergii were purchased from a local farm located in Toucheng Township in Yilan County, Taiwan. After shipping, the prawns were settled at a culture density (total biomass of prawns to water volume) of 1:500. Water temperature in the tank was maintained at approximately $25^{\circ} \mathrm{C}$ using an automated aquarium heater, and dissolved oxygen was provided by continuous air pumping. The hygiene of the aquaculture tanks was scrupulously maintained via daily cleaning, which included removing feed residue from the bottom of the tank, replacing a portion of the water, and feeding the animals on commercial feed twice per day. The animals selected for subsequent experiments were specimens with a carapace length of 30 to $45 \mathrm{~mm}$ and no obvious signs of damage or appendage loss.

\subsection{Electrode assembly}

We considered 3 points in the design of the electrode: (1) preventing short-circuit events during underwater recordings by protecting the connection between the implanted electrode and the external conduction wire through the creation of a chamber using a $2 \mathrm{ml}$ microcentrifuge tube; (2) precisely aligning the 3 electrode leads horizontally, such that the middle one operates as a grounding reference lead, while the other 2 act as signal leads for voltage 

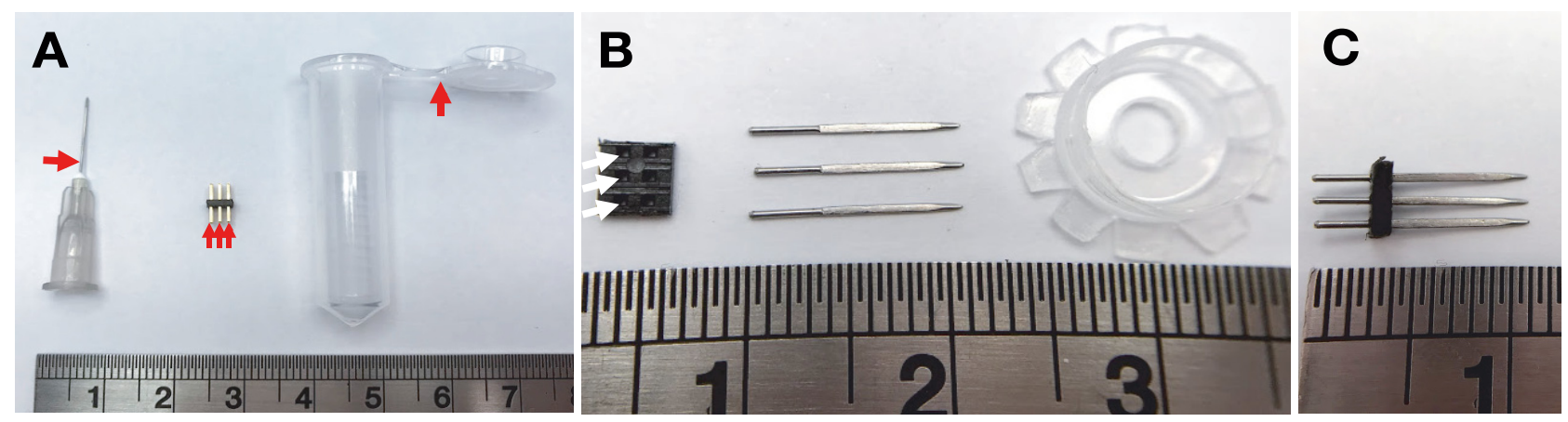

Fig. 1. Proposed 3-channel electrode. (A) Materials used in the construction of the electrode assembly, including (from left to right) a stainless syringe needle (3 needles are required for 1 assembly), a Dupont pin header, and a microcentrifuge tube ( 2 ml size). Red arrows indicate the portion to be sectioned or removed. (B) Dupont pin header with pins removed, 3 syringe needle shafts (cut away from the hub) put into the holes left from the removed pins (indicated by white arrows), and a trimmed cap from a microcentrifuge tube. (C) Pins of female pin header to be removed and replaced by needles

inputs to obtain differential signals; and (3) ensuring that the electrode assembly is as light as possible to avoid placing a physical load (stress) on the animal, with a particular focus on the external conduction wire and quantity of epoxy adhesive.

Fig. 1A presents the materials used in the construction of the electrode assembly, which included three 27 gauge (G) stainless steel syringe needles (to act as leads), the plastic cap cut from a $2 \mathrm{ml}$ microcentrifuge tube (to act as an electrode mounting assembly), a Dupont pin header with $1.27 \mathrm{~mm}$ pitch (to act as the base for the leads), and Coltène/Whaledent dental cement (to mount the needles). The shafts of the needles were cut away from the mounting hub using wire cutters to replace the pins in the Dupont pin header (Fig. 1B). A thin layer of dental cement was used to fix the exposed needles in place within the pin header, after carefully adjusting the length of the shafts to ensure consistency. A hole drilled through the center of the cap was just large enough to allow the 3 leads to pass through. The edges of the cap were cut in a zigzag shape to facilitate adhesion at the end of the assembly process. The electrode assembly was cemented within the cap using a small amount of dental cement, as shown in Fig. 1C. The total cost of the materials was less than US\$1, and even a novice could construct an electrode independently after a $1 \mathrm{~h}$ demonstration.

\subsection{Assembly of external conduction wires}

The materials used to construct the light-weight external conduction wire assembly included 3 sections of thin enameled wire (no. $45 ; 0.07 \mathrm{~mm}$ ), a section of polyethylene tubing (PE20), a single-row male 5-pin header (pitch: $2.54 \mathrm{~mm}$ ), a single-row female 3pin header (pitch: $1.27 \mathrm{~mm}$ ), a section of silicone tubing (no. B07000OD, A-M Systems), a 2 ml microcentrifuge tube, and epoxy glue.

Three sections of enameled wire $(60 \mathrm{~cm}$ in length) were clamped together at both ends using 2 hemostats. The teeth of the hemostats were covered with a short sleeve of silicone tubing (no. 807600, A-M Systems) to prevent damage to the insulation around the wires. The clamped bundle of enameled wires was then hung in a manner that would allow it to droop naturally under the effects of gravity (Fig. 2A). With the upper hemostat held steady, the lower hemostat was rotated counterclockwise to coil the wires tightly together to form a cable, whereupon the lower hemostat was removed. The end of the new cable was then trimmed using scissors and threaded through a PE20 tube until the end extended out of the tube cap by approximately $1 \mathrm{~cm}$.

Both ends of the wire cable were loosened to separate the individual wires, the ends of which were then stripped of their insulation to a distance of approximately $5 \mathrm{~mm}$ (Fig. 2B). One end of each wire was respectively soldered to each of the 3 needles mounted in the female pin header and tube cap assembly (Fig. 2C). The tub cap and connection between the wires and pin header were sealed using epoxy glue (Fig. 2D). A segment of silicon tubing (length $=1 \mathrm{~cm}$ ) was slipped over the other end of the cable (connecting to the amplifier), whereupon the wires were soldered to the middle 3 pins of a 5-pin male pin header and encased within epoxy glue (Fig. 2E). A $2 \mathrm{ml}$ microcentrifuge tube was attached to the epoxysealed cap to make a waterproofed chamber for the electrode connector joint and provide additional buoyancy (Fig. 2F). 


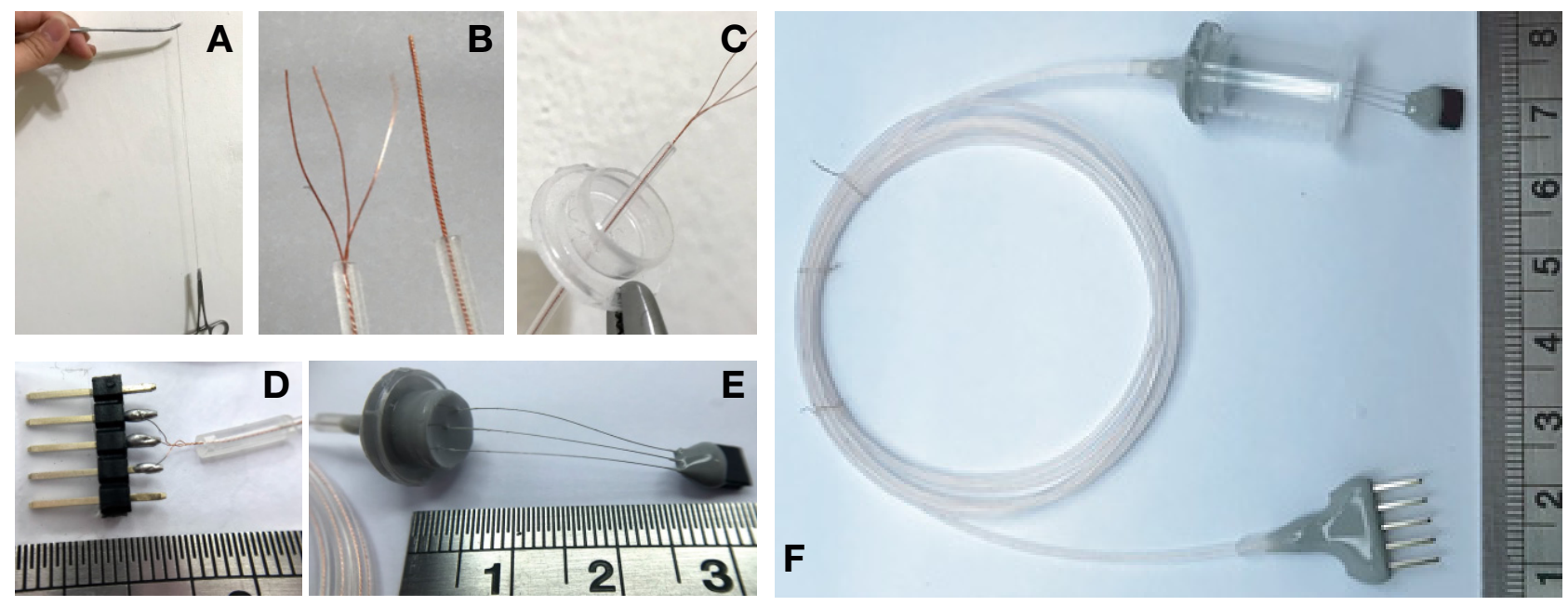

Fig. 2. Materials and steps taken to make the electrocardiogram signal conduction wire. (A) Three enameled wires were clamped on both ends by hemostats, then intertwined to unite them and form a handmade cable. (B) The handmade cable was insulated with a PE20 tube leaving each end exposed (un-insulated) and unwound. (C) A cap from a microcentrifuge tube was used as a plug to seal the chamber shown in (F). A hole was drilled in the centre of the cap to allow the cable to pass through it. (D) The un-insulated ends of the enameled wires were soldered separately to 1 of 3 pins of a 5-pin holder. (E) Joints (and cap) were sealed with epoxy glue (gray color) for waterproofing. (F) The entire handmade cable for ECG signal conduction is shown. The end with a small, female type pin holder and a chamber made with a microcentrifuge tube was connected to the electrodes on the prawn; the other end was connected to the amplifier

\subsection{Electrode implantation}

Based on our previous experience, we determined that needles of roughly $4 \mathrm{~mm}$ in length would be required for prawns with a body weight of 30 to $50 \mathrm{~g}$. The position at which the leads should be implanted is at the posterior edge of a distinct square bump on the carapace, corresponding to the posterior margin of the heart.

With the animal held in place on a clamping frame, a knife was used to scrape the carapace surrounding the heart to facilitate the subsequent adhesion of dental cement. A scalpel was then used to scrape a notch (5 $\mathrm{mm}$ in length) to weaken the carapace. Care must be taken to avoid penetrating the carapace and thereby prevent the loss of excessive body fluids during implantation. The middle lead was aligned with the orientation of the rostrum to ensure accuracy (Fig. 3A).

With the leads held vertically, they were pushed by hand through the notch in the carapace, whereupon the wounds were sealed using a small amount of dental cement. The base of the rostrum was also coated with cement to increase the strength of the attachment (Fig. 3B). After the cement dried, the Dupont female pin header from the external conduction assembly was attached to the male pin header of the electrode, over which was placed the microcentrifuge tube (Fig. 3C).
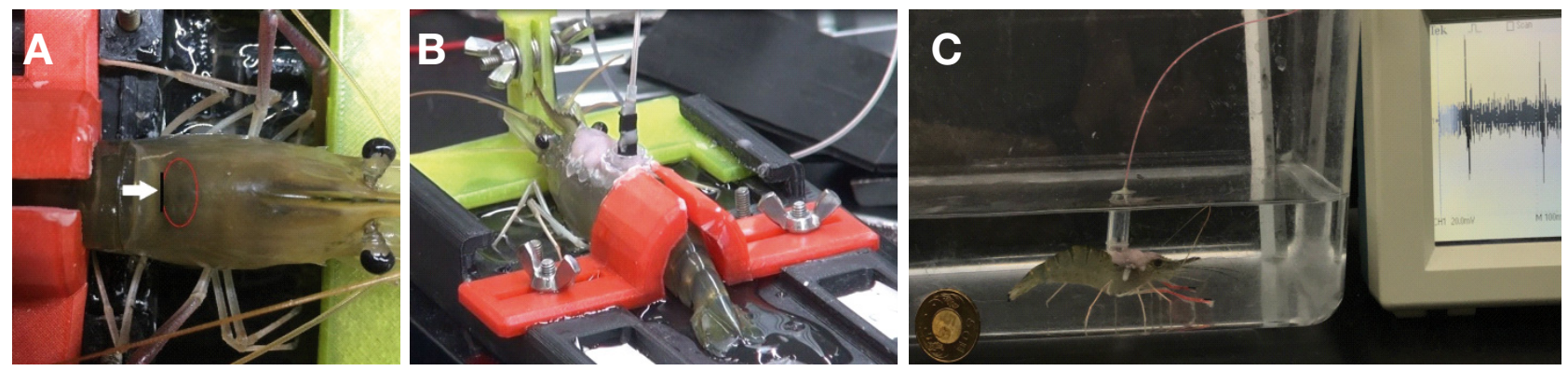

Fig. 3. Implantation of electrocardiogram (ECG) electrode in a giant river prawn. (A) Top view of prawn fixed on frame. The white arrow indicates the notch (black bar) used to locate the site of implantation. The middle of the notch should be aligned with the axis of the rostrum in the carapace (indicated by the white arrow aligned vertically to the notch) to ensure positional accuracy. (B) Insertion site coated with dental cement following electrode implantation. (C) Instance of ECG monitoring of a freely moving giant river prawn $(9.9 \mathrm{~g}$ in weight) after electrode implantation 


\subsection{Data acquisition}

The implanted prawn was moved to the experimental tank (size $30 \times 30 \times 25 \mathrm{~cm}$ ), which was held within a Faraday cage to enable the recording of ECG signals under freely moving conditions. Throughout the recording period, the prawns were fed normally every $12 \mathrm{~h}$. The middle lead was grounded (to act as a reference), and the left and right leads served as voltage inputs to obtain differential signals. The signals were band-pass filtered (approx. 100-300 Hz) and amplified 5000x using a differential amplifier (Model 3000, A-M Systems). After processing, the signals underwent analog-digital conversion using a PowerLab 4/25 (ADInstruments) at a sampling rate of $1000 \mathrm{~Hz}$ before being stored on a computer. Chart 5 software (version 5.5.6; ADInstruments) was used for data analysis.

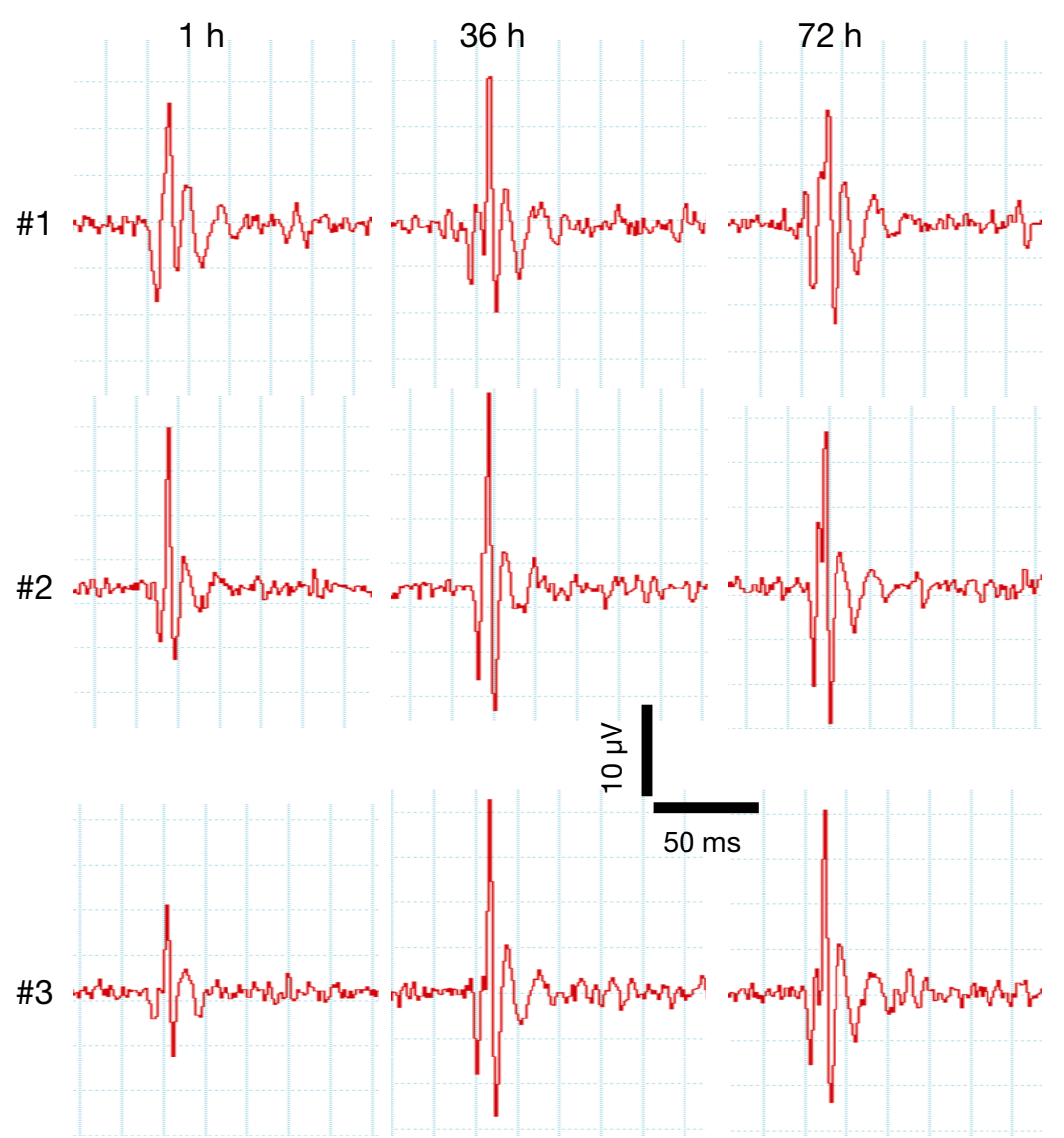

Fig. 4. Stability and comparability of electrocardiogram (ECG) signals recorded using the proposed system. The weights of the 3 prawns were as follows: $41 \mathrm{~g}$ (\#1), $48 \mathrm{~g}(\# 2)$, and $70 \mathrm{~g}(\# 3)$. Data were recorded at 1, 36, and $72 \mathrm{~h}$ after electrode implantation. All of the ECG signals presented similar patterns, with little interday and intersubject variation. Each heartbeat presented 2 distinct larger wavelets followed by a group of smaller wavelets
The contraction of the heart (observed directly through the translucent carapace) produces a group of waveforms in the ECG pattern (see Fig. 4). In calculating the heart rate, the time interval between the largest wavelet in 2 successive waveform groups is treated as the period between 2 heartbeats.

\section{RESULTS}

The proposed ECG recording method proved highly stable, based on the uniformity of results obtained from different animals. Fig. 4 presents the ECG recordings from 3 prawns of various sizes in a freely moving state at 1,36 , and $72 \mathrm{~h}$ after implantation. Clearly, the patterns in the ECG signals did not vary noticeably over time, indicating that the proposed method could be used for analysis over an extended duration.

Fig. 5 presents an example ECG recording obtained over a $38 \mathrm{~d}$ period, showing the ECG intensity (i.e. maximum amplitude of the waveform components) and noise. Despite a noticeable decrease in ECG intensity beginning at $22 \mathrm{~d}$ after implantation, the ECG features remained identifiable with a signal-to-noise ratio exceeding 3.0 .

We also found that changes in ambient temperature had a dramatic effect on the heart rate of 1 prawn in longterm recording. As shown in Fig. 6, the trend between heart rate and ambient temperature remained consistent throughout a 38 d recording period. Under stressful environmental disturbances, we observed temporary cessations of the heartbeat (skipping 1 or more pulses). Manually exchanging the water in the observation tank tended to agitate the prawn, with a result that the frequency of cardiac arrests exceeded the baseline during the first 9 d. Between Days 9 and 20, these events occurred less frequently, indicating that the animal had become acclimated to this interference. During this period, the heart rate of the animal tended to increase during the daytime and decrease when it was dark. After Day 21, this pattern gradually reversed, such that the heart rate dropped during 

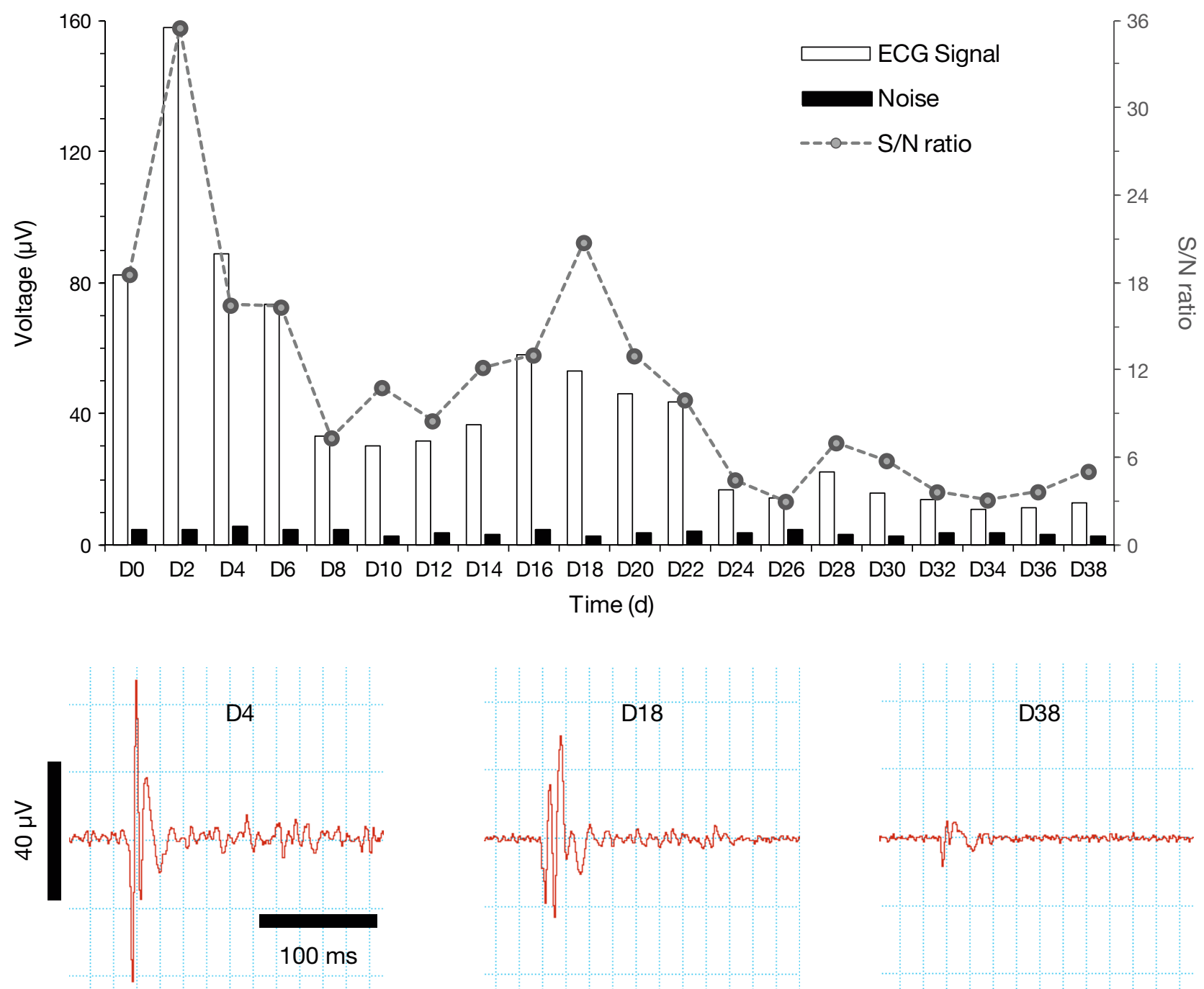

Fig. 5. Electrocardiography data from continuous recording of a giant river prawn $(44 \mathrm{~g})$ over $38 \mathrm{~d}$. The histograms show the amplitude of the electrocardiogram (ECG) signal and background noise during recording, whereas the gray line graph shows the signal-to-noise $(\mathrm{S} / \mathrm{N})$ ratio. The day of electrode implantation is designated D0. Also, signal and noise data were averaged in segments of $2 \mathrm{~d}$. At the time of death on Day 39, the body weight of the prawn was $48 \mathrm{~g}$. The ECG signal began decaying after Day 22; however, the S/N ratio remained above 3.0. The bottom panels show an example of the actual ECG signals recorded on Days 4 (D4), 18 (D18), and 38 (D38) after electrode implantation

the bright period and rose during the dark period. After Day 21, the number of cardiac arrests suddenly increased, while the signal amplitude decreased (Fig. 5). We posit that this can be attributed to a gradual deterioration of the health of the animal, resulting in its death on Day 39, rather than a decline in the recording performance of the electrode.

The implanted electrode was shown not to affect the growth or molting behavior of the prawns. Even during the molting process, the electrode captured ECG signals until the carapace had lifted off entirely. Fig. 7 shows that by Day 10 after molting, there were some anomalies in the appearance of the carapace at the original site of implantation; however, the cara- pace had formed completely with the required hardness. This made it possible to implant electrodes into the same animal a second time, yielding ECG signals with a good signal-to-noise ratio.

The proposed system uses light and easily deformable enameled wires for signal transmission within a PE20 tube to ensure that the apparatus would not affect the movement of the animal. The microcentrifuge tube also provided buoyancy to offset the weight of the other components and thereby reduce physiological stress. As shown in Fig. 8, the continuous recording of ECG patterns for $72 \mathrm{~h}$ revealed no notable increases or decreases in heart rate following electrode implantation. 


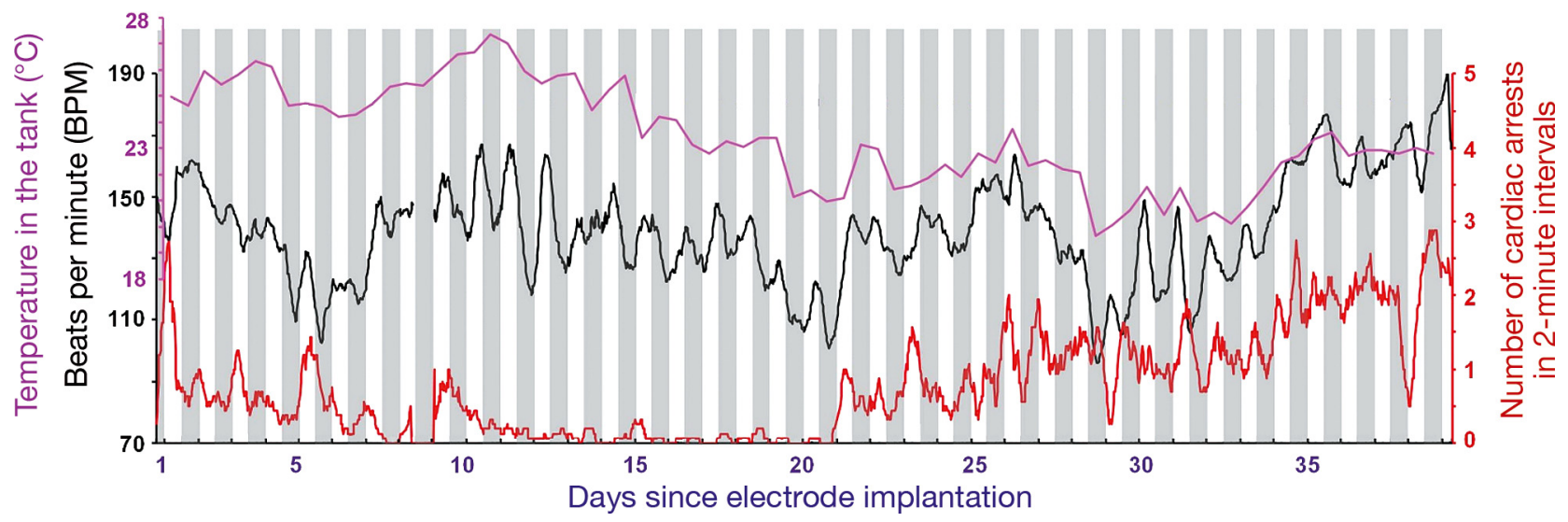

Fig. 6. Changes in the heart rate of a giant river prawn as a function of temperature over $38 \mathrm{~d}$ (same prawn as in Fig. 5). The horizontal axis shows the timeline of this experiment, with white columns indicating light periods and gray columns indicating darkness. The averages of heart rate (black line) and number of cardiac arrests (red line) were calculated from 2 min recording intervals obtained every half hour. These 2 curves are processed by a moving average, which is a calculation used to smooth a curve by creating a series of averages of different subsets of the full data. For each curve, the first element of the moving average is obtained by taking the mean of the initial fixed subset (16 data points) of the original series. Then the data points included in the subset are modified by shifting forward, that is, excluding the first number of the original series and including the next value in the subset. In this way, the calculation continues until the entire data set is averaged
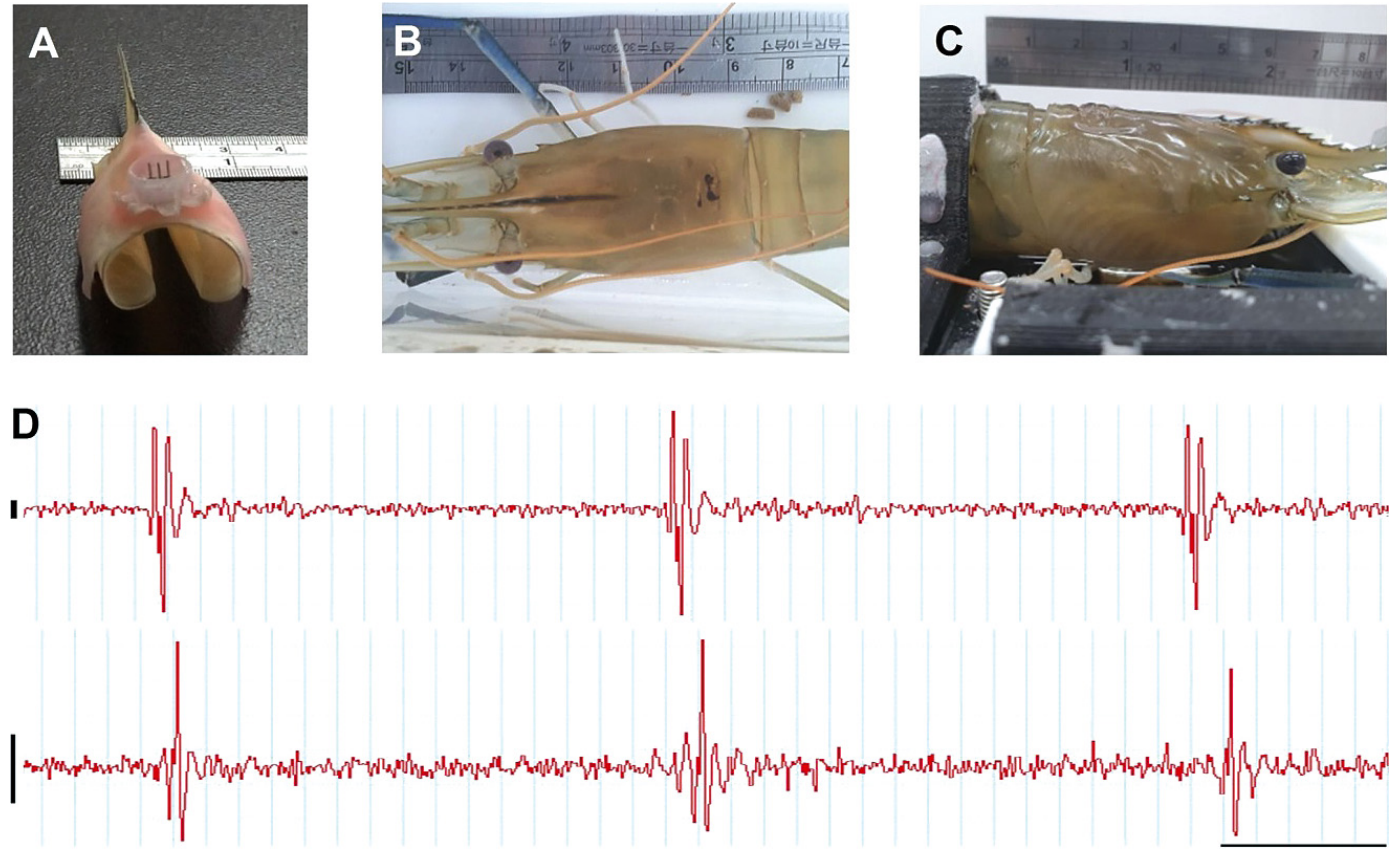

Fig. 7. Giant river prawn (42.4 g) with implanted electrode. (A) Molted cephalothorax carapace section with electrode still attached. (B) Dorsal and (C) lateral views of the prawn $10 \mathrm{~d}$ after molting, showing that the carapace of the prawn was affected by the implanted electrode but still grew to full size. (D) Electrocardiogram (ECG) signals at 2 d prior to molting (upper) and $5 \mathrm{~d}$ after a second electrode was implanted (lower). Scale bar in (D) indicates $10 \mu \mathrm{V}$ vertically and $100 \mathrm{~ms}$ horizontally

\section{DISCUSSION}

Numerous studies have used electrical activity in the myocardium of crustaceans to obtain physiological data, such as heart rate. Importantly, however, electrical activity can be characterized in terms of voltage, impedance, and current, and researchers have yet to attain a consensus as to which of these variables is most representative. Several recent studies have used impedance detectors (Schapker et al. 2002, Bierbower \& Cooper 2009, Weineck et al. 2018, Adams et al. 2019). However, changes in impedance 


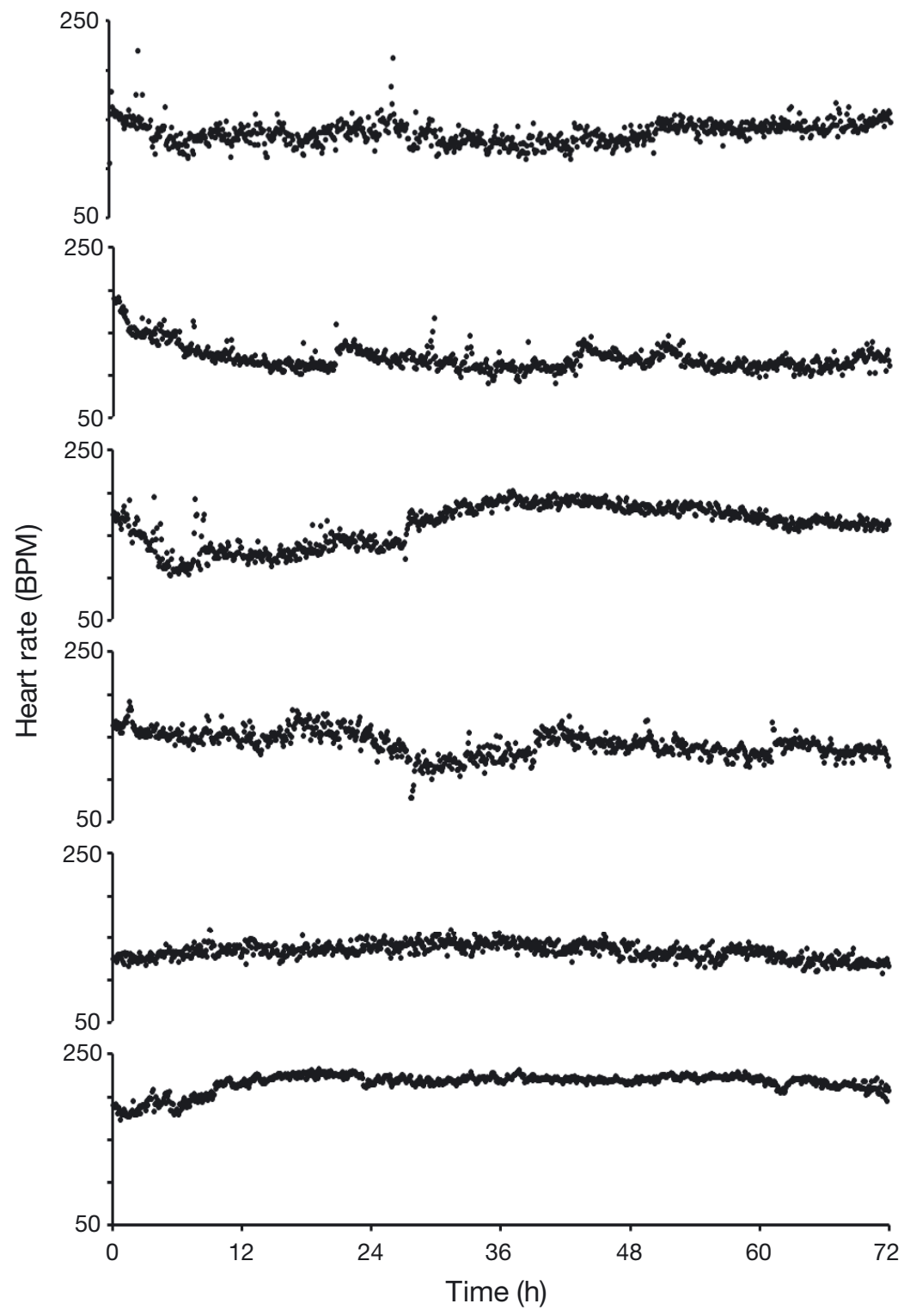

Fig. 8. Heart rate (beats per minute [BPM]) of 6 prawns over $72 \mathrm{~h}$ immediately after electrode implantation. All data were recorded in a bright environment for $72 \mathrm{~h}$

are actually associated with the environment between the 2 leads near the heart, as opposed to changes in membrane potential resulting from depolarization or repolarization; therefore, the impedance signals can only be used to calculate heart rate.

The 2 leads in the studies mentioned above are usually implanted directly above the heart. Thus, the strength of the captured signal depends strongly on the distance between the heart and the non-insulated part of the wire buried within the body. Signal strength also depends on the size of the conductor area used to capture the electrical signal. Unfortunately, previous studies failed to provide details per- taining to crucial variables, such as the length of the wire inserted into the carapace. The heart of a prawn is very close to the inner surface of the carapace, such that inserting a wire of excessive length would likely interfere with the normal beating of the heart. Even if the wire did not come into direct contact with the heart, it would inevitably contact the connective tissue surrounding the organ. A very soft wire would prevent damage to the heart; however, it would also be susceptible to deformation, causing it to extend irregularly across the surface of the heart. Furthermore, it is very difficult to standardize and confirm the distance between the 2 leads, thereby making it difficult to ensure consistency between implant operations.

An ECG measures changes in the membrane potential of the myocardial muscle tissue during each heartbeat, including depolarization and repolarization. Measuring these changes in membrane potential would make it possible to calculate the heart rate as well as other physiological and pathological conditions. Our use of a 3-lead electrode with grounding reference lead and 2 voltage input leads close to the heart was shown to reduce electromagnetic interference from the surrounding environment as well as changes in membrane potential associated with the contraction of surrounding muscle cells of the other organs. The proposed system makes it possible to record the electrophysiological details of heart function, even while the animal is swimming freely. This provides information of far greater richness than can be achieved simply by measuring changes in impedance. In the present study, the electrodes were not implanted directly above the heart but rather were placed close to the posterior side of the heart, thereby preventing damage to heart tissue. The proposed system also makes it possible to control the length of the leads inserted into the carapace, thereby ensuring uniformity across samples.

Nevertheless, there are still a number of questions concerning the standardization of this system. The point of implantation is along the posterior edge of a 
distinct square bump on the surface of the carapace, corresponding to the posterior margin of the heart. Nonetheless, the caudal border of the heart does not necessarily coincide with the square carapace feature in every animal. Thus, during implantation, it is necessary to observe the systole of the heart through the translucent carapace. The margin between the electrode and heart must be small enough to ensure a strong signal but large enough to avoid injuring heart tissue. Ideally, the leads inserted into the prawn should be of sufficient length to reach the desired region adjacent to the heart without any extraneous material, which might otherwise compromise the signalto-noise ratio. Obviously, the size of the heart varies with the size of the body; therefore, the length of the inserted leads must be adjusted according to the size of the test animal. The ideal length can be determined by observing the heart through the translucent carapace from the side. Nonetheless, these observations are only rough approximations; therefore, it is important to remember that operators will tend to differ in terms of electrode placement.

The transparent carapace of giant river prawns makes it possible to observe contractions of the heart directly during the recording of ECG signals. As shown in Fig. 4, each heartbeat provides a corresponding set of waveforms, containing 1 major wavelet (i.e. with the largest amplitude) and 2 minor wavelets (with much smaller amplitudes). Analogous to mammals, these waveforms indicate the changes in potential associated with ventricular depolarization. However, each ventricle in a mammal is connected to only 1 artery, whereas each ventricle in a prawn is connected to 7 arteries (McLaughlin 1983): the unpaired anterior median artery, sternal artery and dorsal abdominal artery, and the paired anterior lateral arteries and hepatic arteries. Furthermore, it is likely that ventricular depolarization in prawns differs from that in mammals, manifesting in multiple QRS complexes.

Invasive recording devices must always be as small and unobtrusive as possible. The electrode structure in the current study uses three $27 \mathrm{G}$ stainless steel needles for insertion into the prawn's body. The diameter of these needles $(0.4 \mathrm{~mm})$ is small enough to minimize damage to the animal, while large enough to resist the rigors of construction and insertion. Materials other than $27 \mathrm{G}$ stainless steel needles (such as microwire) could theoretically be used to fabricate the electrode; however, the wire must be of sufficient strength to penetrate the flexible membranous connective tissue surrounding the heart without the need for carapace laceration, which might otherwise cause uncontrollable bleeding.
Tethering the prawns to a $60 \mathrm{~cm}$ external wire for signal transmission seldom led to entanglement sufficient to hinder the animal's movement. Nonetheless, all assessments were performed with the animal alone in a tank. In the future, it will be necessary to obtain simultaneous recordings from multiple prawns in the same tank. Tethering prawns in this way would make it impossible to conduct multianimal experiments of extended duration or involving excessive movement.

Ideally, the electrodes should be equipped with a wireless transmission system, perhaps mounted on the carapace at the cephalothorax; however, the size and weight of the device would be a key issue. In the external wire system in this study, the connection between the external wires and the output terminal of the electrode was enclosed within a microcentrifuge tube. This made it possible to offset the weight of the tether by taking advantage of the buoyancy provided by the tube. It is very likely that the same approach could be adopted to enclose a wireless transmission system.

Acknowledgements. The authors thank Mr. Jui-Yu Wu for his assistance in completing the experiments in Fig. 7. This work was supported by grants MOST-107-2313-B-197-001 and MOST-106-2621-B-159-001 from the Ministry of Science and Technology of Taiwan.

\section{LITERATURE CITED}

Adams R, Stanley CE, Piana E, Cooper RL (2019) Physiological and behavioral indicators to measure crustacean welfare. Animals (Basel) 9:914

Bierbower SM, Cooper RL (2009) Measures of heart and ventilatory rates in freely moving crayfish. J Vis Exp 32: e1594

Burnovicz A, Oliva D, Hermitte G (2009) The cardiac response of the crab Chasmagnathus granulatus as an index of sensory perception. J Exp Biol 212:313-324

Cooper RM, Schapker H, Adami H, Cooper RL (2011) Heart and ventilatory measures in crayfish during copulation. Open J Mol Integr Physiol 1:36-42

Deshmukh V (2013) Principles of crustacean taxonomy. In: Jose J, Pillai SL (eds) Training programme on taxonomy and identification of commercially important crustaceans of India. Central Marine Fisheries Research Institute, Kochi, p 28-39

Ern R, Huong DTT, Phuong NT, Wang T, Bayley M (2014) Oxygen delivery does not limit thermal tolerance in a tropical eurythermal crustacean. J Exp Biol 217:809-814

Florey E, Kriebel ME (1974) The effects of temperature, anoxia and sensory stimulation on the heart rate of unrestrained crabs. Comp Biochem Physiol A Comp Physiol 48:285-300

* Irisawa H, Irisawa AF (1957) The electrocardiogram of a stomatopod. Biol Bull 112:358-362 
Larimer JL (1962) Responses of the crayfish heart during respiratory stress. Physiol Zool 35:179-186

LListerman LR, Deskins J, Bradacs H, Cooper RL (2000) Heart rate within male crayfish: social interactions and effects of 5-HT. Comp Biochem Physiol A Mol Integr Physiol 125:251-263

McLaughlin PA (1983) Internal anatomy. In: Mantel LH (ed) The biology of Crustacea, Vol. 5. Academic Press, New York, NY, p 1-52

Mickel TJ, Childress JJ (1982) Effects of pressure and temperature on the EKG and heart rate of the hydrothermal vent crab Bythograea thermydron (Brachyura). Biol Bull 162:70-82

Mislin H (1966) Experimenteller Nachweis der Beeinflussung des Elektrokardiogramms (EKG) dekapoder Krebse (Astacus fluviatilis F., Astacus leptodactylus E., Carcinus maenas L.) durch optische Reize (Optocardialer Hemmreflex). Rev Suisse Zool 73:301-312

Editorial responsibility: Bernard Sainte-Marie,

Mont-Joli, Quebec, Canada

Reviewed by: 3 anonymous referees
Schapker H, Breithaupt T, Shuranova Z, Burmistrov Y, Cooper RL (2002) Heart and ventilatory measures in crayfish during environmental disturbances and social interactions. Comp Biochem Physiol A Mol Integr Physiol 131:397-407

* Tsai ML, Hsu WP, Chen TC (2019) Evaluation of suitable temperature range for post-harvest processing of mud crabs through cardiac performance. Aquacult Res 50: 3711-3719

Weineck K, Ray AJ, Fleckenstein LJ, Medley M, Dzubuk N, Piana E, Cooper RL (2018) Physiological changes as a measure of crustacean welfare under different standardized stunning techniques: cooling and electroshock. Animals (Basel) 8:158

* Yazawa T, Katsuyama T (2001) Spontaneous and repetitive cardiac slowdown in the freely moving spiny lobster, Panulirus japonicus. J Comp Physiol A 187 : 817-824

Submitted: June 7, 2020

Accepted: December 11, 2020

Proofs received from author(s): March 23, 2021 